\title{
Sirtuins and diabetes: optimizing the sweetness in the blood
}

\author{
Abhinav Kanwal ${ }^{*^{*}}$ (D) and Liston Augustine Dsouza ${ }^{2}$
}

\begin{abstract}
Diabetes Mellitus (DM) is a chronic disease characterized by elevated levels of glucose in the blood. With time it becomes uncontrollable and invites other complex metabolic diseases. The propensity of the people for this disease is age independent. However, sirtuins, which get activate typically during calorie restriction plays a pivotal role in optimizing effect of blood glucose levels in diabetic patients. Among different sirtuin homologs, some of the sirtuins are known for regulating pathophysiology of diabetic condition. Still the role of other sirtuins in understanding the function and regulatory mechanism in DM is still emerging. In this review, we focused on recent studies which help us to understand about the role of sirtuins and how they regulate the pathophysiology in diabetic condition.
\end{abstract}

Keywords: Sirtuins, Diabetes, Diseases, Mitochondria, Exercise, Deacetylation

\section{Background}

SIRTUINS are the NAD+ dependent enzymes having an ability to alter the acetaylation/deacetylation status of various proteins present in the body. Mammalian sirtuins consists of 7 members, Sirt1-Sirt7, that are differentiated based on their subcellular localization, substrate selectivity etc. (Fig. 1; Table 1). In general, sirtuins are involved in various cellular functions like DNA repair, regulation of transcriptional expression, mitochondrial functioning etc. mainly by altering acetylation/deacetylation status of the proteins by utilizing $\mathrm{NAD}^{+}[1]$. Since the past decade, Sirtuins received much attention because of their involvement in multidisciplinary research areas. This family of enzymes are widely known to regulate aging [2] and diseases including diabetes, heart failure [3, 4], muscle atrophy [5], neurodegenerative disease [6], fibrosis [7] and other metabolic disorders [3]. With the results, researchers around the globe has shown extensive interest to understand the biology of sirtuins, their mechanism of action, mainly their role in lifestyle diseases. Sirtuins are the protein deacetylases with weak mono- ADP ribosyltransferase activity on various histones and transcription factors [8]. They are considered as a switch that balances protein acetylation/

\footnotetext{
*Correspondence: abhinav.kanwal@manipal.edu; abhinavkanwal@gmail.com ${ }^{1}$ Department of Pharmacology, Manipal College of Pharmaceutical Sciences, Manipal Academy of Higher Education, Manipal, Karnataka 576104, India Full list of author information is available at the end of the article
}

deacetylation status and regulates various factors affecting disease conditions associated with energy metabolism, aging and oxidative stress [9]. Initially, most of the studies on sirtuins were focused on cancer biology but now the paradigm is shifted to unveil their role in other disease conditions including diabetes. On the other hand, recent reports have shown that the prevalence of diabetes is increasing globally. With change in lifestyle like increased food consumption and decreased physical activity, diabetes mellitus has been accepted as an epidemic worldwide. The pathophysiology of this chronic metabolic disease is still a mystery despite the advances in diagnostic and therapeutic strategies. Since DM affects both carbohydrate and lipid metabolism, researchers in the recent past have shown the importance of sirtuins as a promising target in fighting the detrimental effects of these disorders. Uncontrolled DM, has terrible consequences on health and wellbeing. In this review, we highlight the role of sirtuins in the pathogenesis of diabetes mellitus and as a target protein in the treatment of DM [10]. Further, research into sirtuin activators is warranted to give a safe and effective DM treatment [11].

\section{Sirtuins functions and localization}

Sirt1 is a first deacetylase of this class and primarily localized in the nucleus. It has prominent role to play in liver, muscles, testis, pancreas, ovary and adipose tissues, 


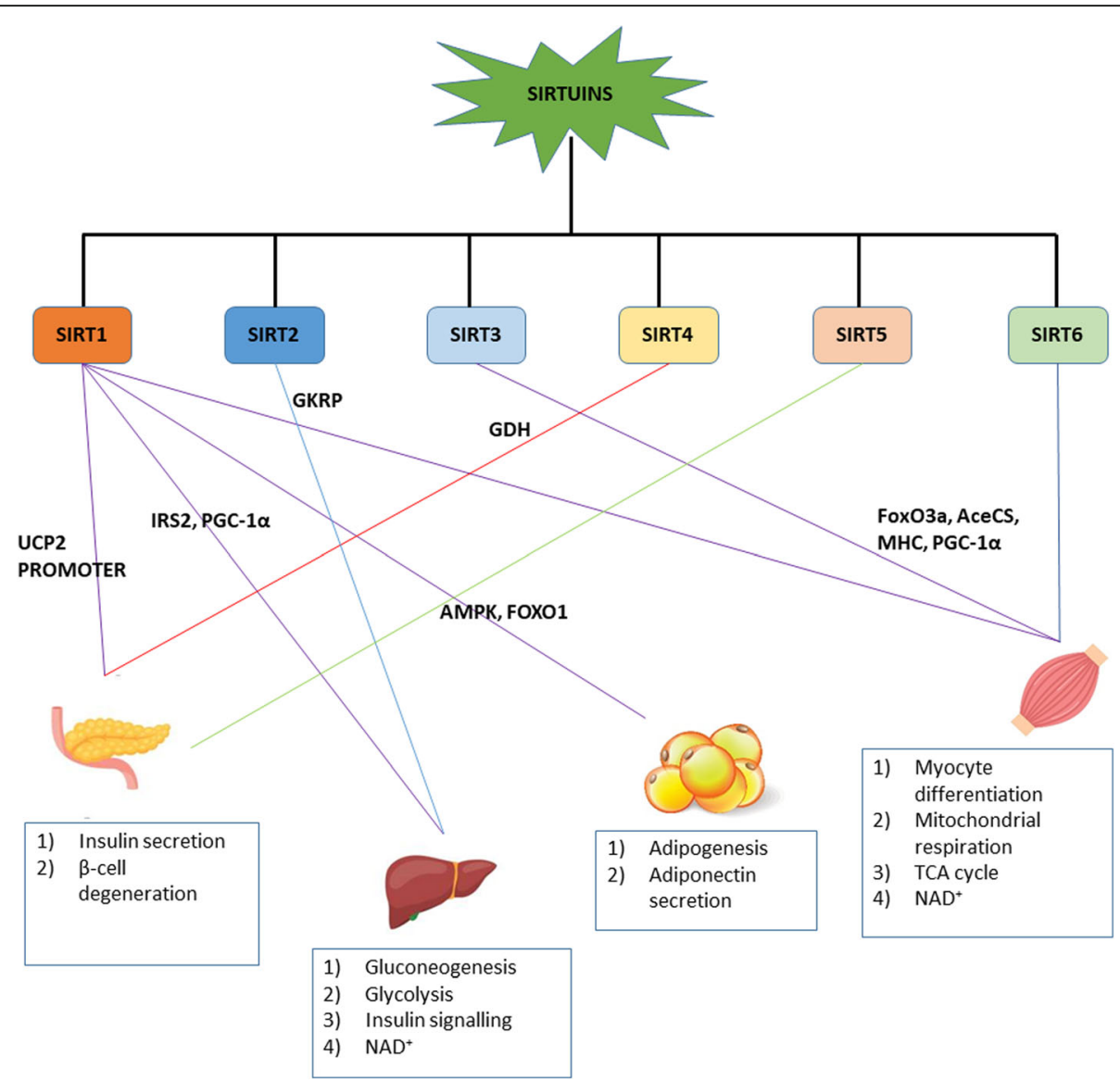

Fig. 1 Role of sirtuins and their functions in different organs in diabetic condition

and it regulates apoptosis, cell proliferation and cell survival. In mammalian cells, calorie restriction activates Sirt1 [12] which reduces the stress induced apoptosis and having a longevity effect. Major substrates for Sirt1 includes FOXO, PGC-1 $\alpha$, androgen receptor, estrogen receptor, $\mathrm{p} 53$, NF- $\mathrm{kB}$ etc. It is involved in various biological functions like Cell survival, metabolism, DNA damage repair, lipid and glucose homeostasis, stress resistance, insulin secretion etc. [1]. Sirt2, the second member of sirtuin isoform is localized in cytoplasm. It

Table 1 Localization of different sirtuins and their enzymatic actions

\begin{tabular}{|c|c|c|c|}
\hline SIRTUINS & FUNCTIONS & LOCALIZATION & ENZYMATIC ACTION \\
\hline$\overline{\text { SIRT1 }}$ & $\begin{array}{l}\text { Cell survival, Metabolism, DNA damage repair, Lipid and glucose } \\
\text { homeostasis, Stress resistance, Insulin secretion, Inflammation, } \\
\text { Neurodegeneration/Axonal degeneration, Apoptosis, Cancer, } \\
\text { Lifespan, Cardiovascular disorder }\end{array}$ & Nucleus, cytosol & Deacetylase \\
\hline SIRT2 & $\begin{array}{l}\text { Control cell cycle, Cell motility/Tumorigenesis, cell differentiation, } \\
\text { Genome stability, Stress response, Neurodegeneration, Metabolism }\end{array}$ & Cytosol, Nucleus & Deacetylase, Demyristoylation \\
\hline SIRT3 & Thermogenesis, Metabolism, Lifespan, Oxidative stress, cell apoptosis & $\begin{array}{l}\text { Mitochondria, } \\
\text { Nucleus }\end{array}$ & Deacetylase \\
\hline SIRT4 & $\begin{array}{l}\text { Insulin secretion, TCA cycle, Fatty acid oxidation, tumour, genome } \\
\text { stability }\end{array}$ & Mitochondria & ADP-ribosylation, deacetylase \\
\hline SIRT5 & $\begin{array}{l}\text { Urea cycle, ketone body synthesis, oxidative stress, cellular } \\
\text { respiration }\end{array}$ & Mitochondria & $\begin{array}{l}\text { Deacetylase, demalonylation, } \\
\text { desuccinylation }\end{array}$ \\
\hline SIRT6 & $\begin{array}{l}\text { DNA repair, glucose homeostasis, Genome stability, Metabolism, } \\
\text { inflammation, cancer, cardiovascular diseases }\end{array}$ & Nucleus & $\begin{array}{l}\text { Deacetylase, ADP-ribosylation, } \\
\text { demyristoylation }\end{array}$ \\
\hline SIRT7 & rRDNA transcription, genome stability, oxidative stress & Nucleus & Deacetylation \\
\hline
\end{tabular}


shows deacetylating activity in the tissues of heart, brain, testes and skeletal muscles. It deacetylates mainly a-tubulin, H4, HOXA10 [13, 14]. The biological actions include regulation of cell cycle and cell motility. It can also deacetylate FOXO transcription factor in response to calorie restriction and oxidative stress [1]. Third member, Sirt3 is a mitochondrial sirtuin and its physiological function involves protein deacetylation in mitochondria of brain, heart, testes, kidney, liver, adipose tissues and muscles. It also increases respiration in mitochondria mainly by stimulating cyclic AMP. Main sirt3 substrates includes, AceCS2, UCP-1, PGC-1a. Sirt3 has been known for longevity in humans [15]. Sirt4, the fourth member of sirtuin family is expressed in almost all tissues, with highest levels found in kidney, heart, brain, pancreas and liver. Like Sirt3, Sirt4 is also localized in mitochondria. Physiologically, it behaves as a regulatory protein in pancreatic $\beta$-cells and liver. It demonstrates ADP-ribosyl transferase activity more efficiently than deacetylase activity. Pull- down studies with Sirt4 identifies insulin degrading enzyme and ADP/ATP carrier proteins, ANT2 and ANT3. It colocalizes with insulin expressing cells in islets of Langerhans [16]. Biological functions of Sirt4 includes secretion of insulin and metabolism by interacting with GDH. Another member, Sirt5 is predominantly a mitochondrial sirtuin, but a significant portion of Sirt5 is localized to cytosol, peroxisomes and nucleus as well. The cytosolic and nuclear proteins exhibit desuccinylation, demalonylation, and deglutarylation. Mitochondrial Sirt5 predominantly exhibit deacetylation and it maintains various cellular and metabolic homeostasis by regulating glucose oxidation, FAO, ammonia detoxification, ketone body formation and ROS management [17]. Another important nuclear deacetylase is Sirt6, which is studied widely for telomeric chromatin. It has shown mono-ADP ribosyltransferase activity and plays significant role in DNA damage repair and ageing. Its main biological functions include DNA damage repair and glucose homeostasis. Lastly, Sirt7 is seen in highly proliferative tissues like spleen, ovary, thyroid, liver and testis. It is absent in tissues of heart, brain and muscle as they are non-proliferative. It is localized in nucleus and known to interact with RNA Polymerase-I and histones. It activates transcription of rRNA and thus involved in regulation of cellular growth, metabolism and cellular survival. A summarized function of all the sirtuins are shown in Table-1.

\section{Sirtuins in diabetes}

Diabetes meliitus was considered as a rare disease where patients had symptoms of frequent urination [18]. Currently, DM is one of the most common disease across the globe causing various other metabolic disorders. It has been estimated that $8.8 \%$ of world's population between the age of 20-79 has DM [19]. Various lifestyle factors including unhealthy food habits, improper health facilities and less physical activity might be the main cause for the increase in diabetic population worldwide $[20,21]$ For this reason, need for the new antidiabetic drug which could be able to target the root cause of the disease is the demand of the time. Sirtuins are emerging class of proteins that are associated with DM and its risk factors. Numerous researchers across the globe are in progress to understand the role and functions of sirtuins in DM and as a novel target in its treatment. Several other studies suggests that physical exercise and calorie restriction can improve the health condition. These can also activate sirtuins which could be beneficial in preventing the progress of the disease. (Figure 1) [11, 22]. Here is a brief description from the current studies reflecting the importance and role of sirtuins in diabetes.

\section{Sirt1}

Sirt1 is the most studied member of class III histone deacetylases known as sirtuins. This maybe because Sirt1 has many roles in cell modulation in cell cycle, mitochondrion metabolism, energy homeostasis, oxidative stress and apoptosis. This isoform has a direct histone deacetylation action, resulting in repression of gene transcription. Alternatively, sirt1 has various metabolic effects by deacetylating non histone proteins like PGC1 $\alpha$, IRS-2, PPAR $\alpha$, PPAR- $\gamma$, mitochondrial uncoupling protein 2 (UCP-2), liver $\mathrm{X}$ factor (LXR), farnesoid $\mathrm{X}$ receptor (FXR) and sterol-regulatory-element binding protein (SREBP). Apart from these functions, Sirt1 also regulate insulin secretion, adipose biogenesis and myogenesis [23]. Activation of Sirt1 plays an important role on glucose/lipid metabolism in Type 2 diabetes mellitus patients. In pancreas, they protect $\beta$-cells and increase insulin secretion. It has role in increasing lipid mobilization in adipose tissues, increases glucose uptake in skeletal muscles and induces mitochondrial biogenesis. These above functions of Sirt1 in relation to DM has been discussed below [24].

Recently Bo et. al. showed that activation of Sirt1 helps in maintaining the redox homeostasis in the diabetic patients. In this study, H3K56Ac levels in diabetic patients were measured. Patients with ages greater than or equal to 40 years, BMI less than $35 \mathrm{~kg} / \mathrm{m} 2$, and patients on diet and/or hypoglycemic agents other than insulin, were recruited. These patients were randomized to once capsule/day of resveratrol $500 \mathrm{mg} /$ day, $40 \mathrm{mg} /$ day and a placebo. It has been observed boosting sirt1 expression through resveratrol treatment led to lower levels of H3K56Ac and also decreased body fat percentage (Fig. 2) [25]. This study described the inverse relationship between the sirt1 expression with H3K56Ac 


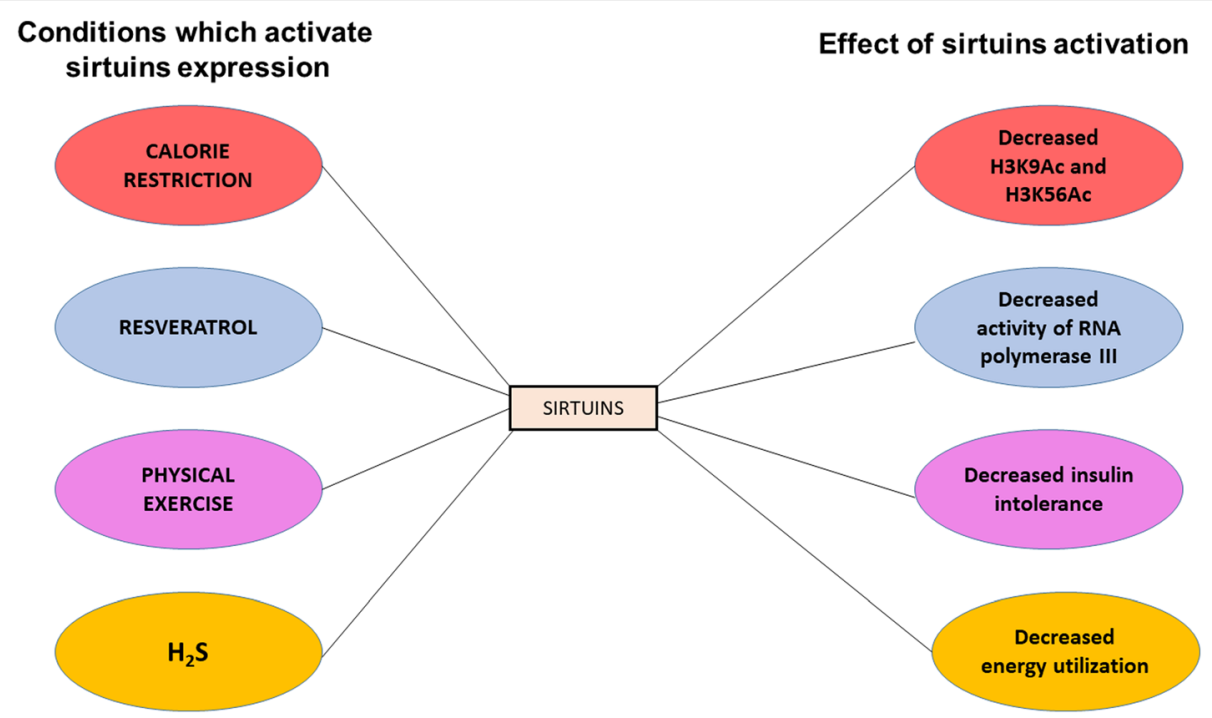

Fig. 2 Factors affecting sirtuin activation and their effects

levels. This confirmed the concept of metabolic memory where addition of acetylated moieties leads to longer effect of high glucose levels among the diabetic patients. Direct relationship of acetylation of proteins with DM or hyperglycemia is confirmed by this study. Diabetic patients have lower levels of sirt1 activity due to excessive mitochondrial fission, resulting in loss of mitochondrial biogenesis [26]. Loss of mitochondrial biogenesis is mediated via acetylation/deacetylation status of PGC- $1 \alpha$ [24]. This means, disturbances in the protein functions should be considered and rectified. Hence, resveratrol, an activator of sirt1 stimulates the sirtuin activity and maintained this homeostasis by deacetylating H3K56Ac.

Liu et. al. described the effect of moderate exercise on $\mathrm{DM}$ induced activation of $\mathrm{NF}-\mathrm{kB}$ and mitochondrial dysfunction. Many studies had shown that NF-kB is an important target required to be suppressed. However, because of crosstalk with many other pathways, the feasibility to work on it is limited. Liu et. al observed inhibition in MuRF-1 and K48-linked polyubiquitination in diabetic mice with moderate exercise and causes reduction in the activation of $\mathrm{I} \kappa \mathrm{B} \alpha / \mathrm{NF}-\mathrm{\kappa} B$ pathway followed by lower levels of IL-6, TNF $\alpha, F 4 / 80$. They reported increased levels of sirt1, PGC1 $\alpha$, AMPK $\alpha$ and mitochondrial complex IV activities due to exercise in diabetic group which upregulates the genes involved in mitochondrial biogenesis [27]. They further confirmed that exercise improves the mitochondrial pathway via Sirt1/AMPK/PGC1-alpha axis. This study provided an insight that different pathways are correlated during complex diabetic condition. Protein degradation is an important phenomenon which offers recycling of the healthy proteins and maintains homeostasis. Among various different mechanisms of protein degradation, ubiquitin-mediated protein degradation is a crucial one. MuRF-1 is the member which belongs to this pathway and helps in maintaining the balance between protein functions. However, it was identified in previous studies that DM causes upregulation of NF-kB and increases the levels of MuRF-1 with the help of FoxO3a. This overall affects the mitochondrial biogenesis process and causes stress by generating reactive oxygen species. Upregulation of sirt1 activity overcomes this condition by inhibiting the degradation pathway and by maintaining the levels of mitochondrial biogenesis proteins [28].

Cardiac complications including cardiomyopathy are common among diabetic patients. Ding. et. al. showed that melatonin prevents Drp-1 mediated mitochondrial fission through Sirt1-PGC-1 $\alpha$ pathway, which in turn is involved in cardiomyopathy. To prove this, they injected mice with streptozocin (STZ) $(50 \mathrm{mg} / \mathrm{kg} /$ day $)$ in citrate buffer i.p for 5 consecutive days. Mice with fasting glucose levels greater than $11.1 \mathrm{mmol} / \mathrm{L}$ were considered to have DM. These mice were treated with melatonin (10 $\mathrm{mg} / \mathrm{kg}$, once daily, i.p) or mdivi-1. The results showed that melatonin prevents Drp-1 mediated mitochondrial fission through Sirt1-PGC1 $\alpha$ and thus alleviating cardiac dysfunction in diabetic mice. Melatonin treatment increases sirt1 expression at both in-vitro and in-vivo studies. Thus, indicating that melatonin role in mitochondrial dynamics could be a novel target as a cardio protective agent in diabetic patients.

\section{Sirt2}

Sirt2, the second member of human sirtuin family, is crucial for hepatic glucose uptake (HGU) [29]. Sirt2 has mostly been implicated for its role in regulating adipocytes tissue development and its functions. Beyond its 
role in regulating adipocytes development, Sirt2 also plays an important role in maintaining metabolic homeostasis. Glucose homeostasis is controlled, either by facilitating hepatic glucose uptake or promoting phosphoenolpyruvate carboxykinase (PEPCK1) degradation which in turn inhibits gluconeogenesis [29-31]. Hepatic glucose uptake controls postprandial hyperglycemia, as impairment can result into higher glucose levels. HGU in turn is regulated by glucokinase and glucose-6-phosphatase. Glucokinase is an enzyme that acts as a catalyst in phosphorylation of glucose to glucose-6-phosphate, and also another enzyme glucose-6-phosphotase catalyzes the reverse of the above reaction. Hence HGU is dependent on the balance between G6Pase and Glucokinase. With an acute increase in glucokiase activity, postprandial blood glucose level increases, and this is induced by a glucokinase regulatory protein after post translational modifications. Role of sirt2 on HGU mediated by glucokinase regulatory protein (GKRP) is shown by Wananabe et. al. They showed that GKRP acetylation have an inverse relationship with sirt2 expression and sirt2 directly affects the hepatic glucose uptake. Absence of sirt2 in the liver of non-diabetic mice causes glucose intolerance. Similarly, sirt2 overexpression in diabetic mice mitigates impaired glucose tolerance and promotes hepatic glucose uptake by deacetylating K126 of GKRP [29]. They had proved the importance of NAD+ /Sirt2 in hepatic glucose uptake. They revealed that sirt2 is a crucial member having deacetylase activity and the activity diminishes with decrease in liver NAD+ levels. This condition corresponds to higher GKRP acetylation at K126 that leads to the impairment of hepatic glucose uptake. This further leads to insulin resistance and proves that GKRP could be used as a potential therapeutic target in DM or insulin resistance. Apart from HGU dependent glucose homeostasis, Sirt2 prevents ubiquitylation dependent PEPCK degradation via deacetylation. PECPK is an enzyme that catalyzes the rate-limiting irreversible step of gluconeogenesis. PEPCK is an important marker in the evaluation of T2DM. Lys70, Lys71, and Lys594 of human PEPCK1 was found to be acetylated which led to decreased stability of these proteins leading to reduced protein levels. In a study, it was found that Sirt2 deacetylates PEPCK1 and stabilizes the protein by preventing its degradation. Thus it maintains the glucose homeostasis [30, 32].

\section{Sirt3}

Third homolog, Sirt3 is an $\mathrm{NAD}^{+}$dependent mitochondrial sirtuin which improves mitochondrial health by controlling its dynamics. Sirt3 is a novel regulator of mitochondrial function, Insulin signaling and insulin resistance in DM [33]. It has been shown to direct mitochondrial respiration and its substrates include subunits of respiratory chain complex $[9,34]$. It has been implicated to cause metabolic diseases in humans and rodents. Sun et. al. illustrates the importance of exogenous $\mathrm{H}_{2} \mathrm{~S}$ and sirt3 in heart tissue of $\mathrm{db} / \mathrm{db}$ mice. They showed that exogenous $\mathrm{H}_{2} \mathrm{~S}$ increases the sirt3 expression by maintaining the $\mathrm{NAD}^{+} / \mathrm{NADH}$ ratio (Fig. 2). It maintains acetylation level and different activities of the enzymes involved in the energy metabolism inside the cell. Ultimately, this is done by upregulating the sirt3 levels by controlling and maintaining the deacetylation status [35]. In addition, it had shown that Sirt3 KO-mice exhibited insulin resistance due to increased levels of protein acetylation in skeletal muscles. Sirt3 activity also found to be decreased in skeletal muscle models of DM. Since, Sirt3 is involved with functions of mitochondria, it is important to understand the involvement of sirt3 in the regulation of metabolism to establish a link between mitochondrial function and metabolic disease. Similar to Sirt1, streptozotocin induced diabetic mice, suggested that lower expression of Sirt3 resulted in impaired insulin signaling. A study found that $\mathrm{C} 2 \mathrm{C} 12$ myoblasts with Sirt3 knockdown showed marked insulin resistance with decrease in IGF-1 stimulated IRS-1 tyrosine phosphorylation, with a parallel decrease in phosphorylation of Akt and MAPK. This was independent of insulin induced tyrosine phosphorylation on insulin receptor, but led to marked increase in JNK phosphorylation. The increased JNK phosphorylation was due to oxidative stress caused by Sirt3 knockdown. Targeted deletion of Sirt3 in skeletal muscles led to decrease in insulin signaling, suggesting Sirt3 is an important regulator of insulin signaling in muscles and alterations in expression can lead to insulin resistance [33].

\section{Sirt4}

Sirt4 is another isomer of human sirtuins that is NAD dependent protein lipoamidase, ADP-ribosyl transferase and deacetylase. It catalyzes removal of lipoyl- and biotinyl- more efficiently than acetyl lysine and inhibits pyruvate dehydrogenase (PDH) activity in a phosphorylation independent manner [36]. Wood et. al. described the role sirt4 in metabolic homeostasis. They showed that sirt4 knockout Drosophila flies were short-lived and unable to utilize energy reserves during starvation, which ultimately leads to death. This might have happened because of defects in their metabolic pathways [37]. Sirt4 acts as a metabolic regulator and involves in glucose metabolism by interacting with glutamate dehydrogenase $(\mathrm{GDH})$ by converting glutamate to $\alpha$-ketoglutaric acid in mitochondrion $[38,39]$. It represses fatty acid oxidation and promotes lipid anabolism by inhibiting malonylCoA-decarboxylase (MCD) [40]. GDH is associated with metabolism of glutamate and glutamine and promotes secretion of insulin via generation of ATP. Sirt4 represses GDH, and hence inhibits insulin secretion. It has been shown that amino acid induced secretion of insulin 
was increased due to loss of Sirt4 [41]. This similar effect was seen in the pancreatic $\beta$-cells of Sirt4 knockout mice, where these mice were progressively developed glucose intolerance and insulin resistance. These findings showcase Sirt4 as an important regulator in maintaining insulin secretion and glucose homeostasis. Sirt 4 has also shown to have Mono-ADP-ribosylate activity. It interacts with insulin degrading enzyme and ANT2 and ANT3 subunit of ATP/ADP translocate in pancreatic $\beta$ cells. This ultimately downregulates the glucose mediated insulin secretion. It also catalyze $\mathrm{N}$-acetyl-lysine deacetylation of malonylCoA decarboxylase, an enzyme that catalyzes conversion of malonyl-CoA to acetyl CoA and involved in downregulation of fatty acid oxidation in muscles. Sirt 4 was further found to catalyze delipoylation of N-lipoyl-lysine of pyruvate dehydrogenase thus inhibits the activity of the complex. This Sirt4 catalyzed delipoylation of PDH complex is involved in metabolic flux and switched to glutamine as a carbon source for citric acid cycle. This highlights the importance of Sirt4 in regulation of metabolism via inhibiting PDH complex activity [42]. Sirt4 also has protecting role against diabetic nephropathy. Diabetic nephropathy is a complication of DM and associated with capillary damage and increased mortality. Jian. et. al showed the association of Sirt4 and diabetic nephropathy in a glucose induced mice podocyte model. They have shown that Sirt4 overexpression increases proliferation and suppressed apoptosis. This is accompanied by increase in mitochondrial membrane potential and reduced production of ROS. Increased sirt4 expression downregulated the expression of apoptotic proteins like NOX1, Bax and phosphorylated p38 and upregulates $\mathrm{Bcl}-2$ expression in glucose stimulated podocytes. These findings proves that Sirt4 overexpression prevents glucose induced podocyte apoptosis and ROS production in diabetic nephropathy [43].

\section{Sirt5}

Sirt5 is yet another mitochondrial sirtuin which is less explored. It possesses weak deacetylase activity but have deglutarylation, desuccinylation, demalonylation activities on lysine residues. Current studies demonstrate that sirt5 activity is increased in DM patients [44]. This reveals that increased sirt5 activity has a positive impact on age and blood glucose. Studies have identified that sirt5 is involved in ketone body synthesis, TCA cycle, $\beta$-oxidation of fatty-acids, amino acid catabolism, glycolysis and ATP synthesis $[45,46]$. Recently, a study involved two pancreatic $\beta$-cell lines MIN6 and INS-1 has shown that inhibition of Sirt5 promoted proliferation of pancreatic $\beta$-cells and secretion of insulin [44]. However previous studies have shown that sirt 5 expression facilitates cancer cell proliferation. Nevertheless, these contradicting results suggested that since cancer and diabetes being two different disease conditions, there could be many factors contributing to different functions of Sirt5, including metabolism, cellular microenvironment and activation of oncogene. Hence therapeutic benefits of sirt5 remains unclear.

\section{Sirt6}

Exploring further, the importance of sirtuins in diabetic conditions, other analogue of sirtuin called sirt6 is important for maintaining glucose homeostasis and insulin sensitivity. Sirt6 deficiency causes glucose intolerance and impaired glucose-stimulated insulin secretion. Qin et. al. had shown that Sirt6 plays an important role in the normal functioning of $\beta$-cell via deacetylation of histone $\mathrm{H} 3$ and plays a critical role in maintaining its functions and viability. $\beta$-cells of pancreas showed decreased sensitivity when Sirt6 is inhibited. Sirt6 deficiency increases H3K9Ac, H3K56Ac levels and active RNA polymerase II at the promoter region of Txnip, causing expression of Txnip that promotes $\beta$-cell apoptosis [47].

\section{Sirtuins as future anti-diabetics}

Prevalence of diabetes mellitus is growing worldwide, with majority of cases belong to T2DM. India ranks second globally in terms of diabetes prevalence in adults. Currently available classes of antidiabetic agents are used alone or in combination but rarely achieve treatment targets. A large number of molecules, some with novel mechanisms are in pipeline [48]. Biological anti-diabetics have been widely studies and developed in past few decades. New chemical antidiabetic agents are very few that patients with advanced DM still dependent on insulin. In the recent past, several studies suggests that acetylation is a conservative protein modification and that regulation of glucose metabolism enzymes may affect gluconeogenesis. This paves way to develop anti-diabetic drugs that regulate protein acetylation. Since sirtuins regulate the acetylation/deacetylation status of certain proteins, the potential role of sirtuins for effective treatment or prevention of DM has resulted in significant efforts in finding novel therapeutics that can activate sirtuins [48]. The conditions which activates sirtuins and its effects are shown in Fig. 2. Among the activators of sirtuins, resveratrol is most widely studied. It has been studied that mice experiment that resveratrol had many antidiabetic effects on rodents fed a high fat diet, had improved glucose tolerance and increased insulin sensitivity. When the metabolic effects of resveratrol were studied in animal and humans, it was evident that it had little effect on metabolism in nondiabetic patients, hence reducing the risk of hypoglycemia. Apart from resveratrol, development of many effective sirtuin activators is currently underway. Recently, AS101, a tellurium compound, that increases Sirt1 expression and activity 
shows a promising treatment for DM [49]. SRT1720, is a small molecule Sirt1 activator, which is 1000 times more potent than resveratrol. In a mice experiment it was seen that, 10 weeks treatment led to reduced post prandial glucose levels, indicating potential to treat Type2 DM [50]. The therapeutic strategies of activating sirtuins is mainly focused on Sirt1 because it is most widely studied sirtuin. However other strategies of activating Sirt1, Sirt2, Sirt3, Sirt4, Sirt6 are yet to be explored [11].

\section{Conclusions}

A number of evidence had shown the involvement of epigenetic regulation in different metabolic and vascular diseases. Like many other diseases, DM also showed the epigenetic variation in different human tissues. Hence, Importance of understanding the physiological and functional role of sirtuins can be understood by the fact that lot of debate is going on to find out and establish the potential epigenetic biomarker to identify the early risk and progression of DM. Sirtuins are the crucial sensors present in different compartments of the cell and act as an important epigenetic regulator. Understanding of the mechanisms through which sirtuins function during diabetic condition is indispensable. Since past decade many new studies have been published which prompt us to dig further deeper to explore. Among seven sirtuins, sirt1 \& sirt3 are widely studied in comparison to others. They maintain and regulates the acetylation status of different modified proteins in various disease conditions including DM. Hence, it acts as an important epigenetic switch which controls the normal functioning of several targets and prevent them to function in an odd manner. Lower levels of sirtuins disturbs various mechanisms including mitochondrial homeostasis, ROS production, increased inflammation markers and cause acetylation of numerous proteins that affects the signaling cascades. Hence, it is very important for us to understand how these sirtuins functions mechanistically and can be used therapeutically for the treatment in the patients suffering from DM or diabetes induced metabolic disorders.

\section{Acknowledgements}

Not applicable.

\section{Funding}

No funding source is available.

\section{Availability of data and materials}

Data sharing not applicable to this article as no datasets were generated or analysed during the current study.

\section{Authors' contributions}

The review article was envisaged by AK, the relevant researches were analyzed by LAD. The manuscript was edited by AK. Both authors read and approved the final manuscript.

Ethics approval and consent to participate Not applicable.
Consent for publication

Not applicable.

\section{Competing interests}

The authors declare that they have no competing interests.

\section{Publisher's Note}

Springer Nature remains neutral with regard to jurisdictional claims in published maps and institutional affiliations.

\section{Author details}

${ }^{1}$ Department of Pharmacology, Manipal College of Pharmaceutical Sciences, Manipal Academy of Higher Education, Manipal, Karnataka 576104, India. ${ }^{2}$ Manipal College of Pharmaceutical Sciences, Manipal Academy of Higher Education, Manipal, Karnataka 576104, India.

Received: 26 November 2018 Accepted: 29 January 2019

Published online: 09 February 2019

\section{References}

1. Shoba B, Lwin ZM, Ling LS, Bay BH, Yip GW, Kumar SD. Function of sirtuins in biological tissues. Anatomical record (Hoboken, N.J. : 2007), 292(4), 536543 (2009).

2. Khan RI, Nirzhor SSR, Akter R. A review of the recent advances made with SIRT6 and its implications on aging related processes, major human diseases, and possible therapeutic targets. Biomolecules. 2018;8(3):44.

3. Katsuumi G, Shimizu I, Yoshida Y, Minamino T. Vascular senescence in cardiovascular and metabolic diseases. Frontiers in cardiovascular medicine. 2018;5:18-8.

4. Pillai VB, Kanwal A, Fang YH, et al. Honokiol, an activator of Sirtuin-3 (SIRT3) preserves mitochondria and protects the heart from doxorubicin-induced cardiomyopathy in mice. Oncotarget. 2017:8(21):34082-98.

5. Samant SA, Kanwal A, Pillai VB, Bao R, Gupta MP. The histone deacetylase SIRT6 blocks myostatin expression and development of muscle atrophy. Sci Rep. 2017;7(1):11877-7

6. Jeśko H, Wencel P. Strosznajder RP. Strosznajder JB. Sirtuins and their roles in brain aging and neurodegenerative disorders. Neurochem Res. 2017; 42(3):876-90.

7. Bindu S, Pillai VB, Kanwal A, et al. SIRT3 blocks myofibroblast differentiation and pulmonary fibrosis by preventing mitochondrial DNA damage. American journal of physiology. Lung cellular and molecular physiology. 2017;312((1)):L68-78.

8. Nakagawa T, Guarente L. Sirtuins at a glance. J Cell Sci. 2011;124(6):833-8.

9. Kanwal A. Functional and therapeutic potential of mitochondrial SIRT3 deacetyalse in disease conditions. Expert review of clinical pharmacology, (2018).

10. Turkmen K, Karagoz A, Kucuk A. Sirtuins as novel players in the pathogenesis of diabetes mellitus. World J Diabetes. 2014;5(6):894-900.

11. Huynh FK, Hershberger KA, Hirschey MD. Targeting sirtuins for the treatment of diabetes. Diabetes management (London, England), 3(3), 245257 (2013).

12. Cantó C, Auwerx J. Caloric restriction, SIRT1 and longevity. Trends Endocrinol Metab. 2009:20(7):325-31.

13. Vaquero A, Scher MB, Lee DH, et al. SirT2 is a histone deacetylase with preference for histone H4 Lys 16 during mitosis. Genes Dev. 2006;20(10): 1256-61.

14. de Oliveira RM, Sarkander J, Kazantsev AG, Outeiro TF. SIRT2 as a therapeutic target for age-related disorders. Front Pharmacol. 2012;3:82-2.

15. Kincaid B, Bossy-Wetzel E. Forever young: SIRT3 a shield against mitochondrial meltdown, aging, and neurodegeneration. Front Aging Neurosci. 2013:5:48-8.

16. Ahuja N, Schwer B, Carobbio S, et al. Regulation of insulin secretion by SIRT4, a mitochondrial ADP-ribosyltransferase. J Biol Chem. 2007;282(46): 33583-92

17. Kumar S, Lombard DB. Functions of the sirtuin deacylase SIRT5 in normal physiology and pathobiology. Crit Rev Biochem Mol Biol. 2018;53(3):311-34.

18. Alonso-Magdalena P, Ropero AB, Soriano S, Quesada I, Nadal A. Bisphenol-a: a new diabetogenic factor? Hormones (Athens, Greece), 9(2), 118-126; 2010.

19. Ogurtsova K, da Rocha Fernandes JD, Huang Y et al. IDF Diabetes Atlas: Global estimates for the prevalence of diabetes for 2015 and 2040. Diabetes Research and Clinical Practice, 128, 40-50). 
20. Riccardi G, Vitale M, Giacco R. Treatment of Diabetes with Lifestyle Changes: Diet. In: Bonora E, DeFronzo R, editors. Diabetes. Epidemiology, Genetics, Pathogenesis, Diagnosis, Prevention, and Treatment: Springer International Publishing, Cham; 2018. p. 1-16.

21. 4. Lifestyle Management: <em>Standards of Medical Care in Diabetes-2018</ em>. Diabetes Care, 41 (Supplement 1), S38-S50 (2018).

22. Samant SA, Kanwal A, Pillai VB, Bao R, Gupta MP. The histone deacetylase SIRT6 blocks myostatin expression and development of muscle atrophy. Sci Rep. 2017;7(1):11877

23. Guclu A, Erdur FM, Turkmen K. The Emerging Role of Sirtuin 1 in Cellular Metabolism, Diabetes Mellitus, Diabetic Kidney Disease and Hypertension. Experimental and clinical endocrinology \& diabetes : official journal, German Society of Endocrinology [and] German Diabetes Association, 124(3), 131139 (2016).

24. Kitada M, Koya D. SIRT1 in type 2 diabetes: mechanisms and therapeutic potential. Diabetes \& metabolism journal. 2013;37(5):315-25.

25. Bo S, Togliatto G, Gambino R, et al. Impact of sirtuin-1 expression on H3K56 acetylation and oxidative stress: a double-blind randomized controlled trial with resveratrol supplementation. Acta Diabetol. 2018;55(4):331-40.

26. Tang BL. Sirt1 and the mitochondria. Molecules and cells. 2016;39(2):87-95.

27. Liu H-W, Chang S-J. Moderate exercise suppresses NF-KB signaling and activates the SIRT1-AMPK-PGC1a Axis to attenuate muscle loss in diabetic db/db mice. Front Physiol. 2018;9:636.

28. Chalkiadaki A, Igarashi M, Sebastian Nasamu A, Knezevic J, Pershing GL. Muscle-specific SIRT1 gain-of-function increases slow-twitch fibers and ameliorates pathophysiology in a mouse model of Duchenne muscular dystrophy; 2014.

29. Watanabe H, Inaba Y, Kimura K, et al. Sirt2 facilitates hepatic glucose uptake by deacetylating glucokinase regulatory protein. Nat Commun. 2018;9(1):30.

30. Zhang M, Pan Y, Dorfman RG, et al. Sirtinol promotes PEPCK1 degradation and inhibits gluconeogenesis by inhibiting deacetylase SIRT2. Sci Rep. 2017; 7(1):7.

31. Giblin W, Skinner ME, Lombard DB. Sirtuins: guardians of mammalian healthspan. Trends in genetics : TIG. 2014;30(7):271-86.

32. Giblin W, Lombard DB. Chapter 3 - Sirtuins, Healthspan, and Longevity in Mammals. In: Handbook of the Biology of Aging (Eighth Edition). Kaeberlein, MR, Martin, GM (Eds.) (Academic Press, San Diego, 2016) 83-132.

33. Jing E, Emanuelli B, Boucher J, Saha A, Ruderman N, Kahn C. Sirt3, a novel regulator of mitochondrial function and insulin signaling in diabetes and insulin resistant states; 2010.

34. Ansari A, Rahman MS, Saha SK, Saikot FK, Deep A, Kim K-H. Function of the SIRT3 mitochondrial deacetylase in cellular physiology, cancer, and neurodegenerative disease. Aging Cell. 2017;16(1):4-16.

35. Sun Y, Tian Z, Liu N, et al. Exogenous H2S switches cardiac energy substrate metabolism by regulating SIRT3 expression in db/db mice. J Mol Med. 2018; 96(3):281-99.

36. Mathias RA, Greco TM, Oberstein A, et al. Sirtuin 4 is a lipoamidase regulating pyruvate dehydrogenase complex activity. Cell. 2014;159(7):1615-25.

37. Wood JG, Schwer B, Wickremesinghe PC, et al. Sirt4 is a mitochondrial regulator of metabolism and lifespan in Drosophila melanogaster. Proc Natl Acad Sci. 2018;115(7):1564-69.

38. Fernandez-Marcos PJ, Serrano M. Sirt4: the glutamine gatekeeper. Cancer Cell. 2013;23(4):427-8.

39. Plaitakis A, Kalef-Ezra E, Kotzamani D, Zaganas I, Spanaki C. The glutamate dehydrogenase pathway and its roles in cell and tissue biology in health and disease. Biology. 2017;6(1):11.

40. Pannek M, Simic Z, Fuszard M, et al. Crystal structures of the mitochondrial deacylase Sirtuin 4 reveal isoform-specific acyl recognition and regulation features. Nat Commun. 2017;8(1):1513.

41. Haigis MC, Mostoslavsky R, Haigis KM, et al. SIRT4 inhibits glutamate dehydrogenase and opposes the effects of calorie restriction in pancreatic beta cells. Cell. 2006;126(5):941-54.

42. Li S, Zheng W. Chapter Five - Mammalian Sirtuins SIRT4 and SIRT7. In: Progress in molecular biology and translational science. Zheng, W (Ed. (Academic Press, 2018) 147-168.

43. Shi J-X, Wang Q-J, Li H, Huang Q. SIRT4 overexpression protects against diabetic nephropathy by inhibiting podocyte apoptosis. Experimental and therapeutic medicine. 2017:13(1):342-8.

44. Ma Y, Fei X. SIRT5 regulates pancreatic $\beta$-cell proliferation and insulin secretion in type 2 diabetes. Experimental and therapeutic medicine. 2018; 16(2):1417-25.
45. Koronowski KB, Khoury N, Morris-Blanco KC, Stradecki-Cohan HM, Garrett TJ, Perez-Pinzon MA. Metabolomics based identification of SIRT5 and protein kinase C epsilon regulated pathways in brain. Front Neurosci. 2018;12:32-2.

46. Koronowski KB, Khoury N, Morris-Blanco KC, Stradecki-Cohan HM, Garrett TJ, Perez-Pinzon MA. Metabolomics Based Identification of SIRT5 and Protein Kinase C Epsilon Regulated Pathways in Brain. Frontiers in Neuroscience. 2018;12((32))

47. Qin K, Zhang N, Zhang Z et al. SIRT6-mediated transcriptional suppression of Txnip is critical for pancreatic beta cell function and survival in mice (2018).

48. Chikara G, Sharma PK, Dwivedi P, Charan J, Ambwani S, Singh S. A narrative review of potential future antidiabetic drugs: should we expect more? Indian journal of clinical biochemistry : IJCB. 2018;33(2):121-31.

49. Halperin-Sheinfeld M, Gertler A, Okun E, Sredni B, Cohen HY. The tellurium compound, AS101, increases SIRT1 level and activity and prevents type 2 diabetes. Aging. 2012;4(6):436-47.

50. Mitchell SJ, Martin-Montalvo A, Mercken EM, et al. The SIRT1 activator SRT1720 extends lifespan and improves health of mice fed a standard diet. Cell Rep. 2014;6(5):836-43

\section{Ready to submit your research? Choose BMC and benefit from:}

- fast, convenient online submission

- thorough peer review by experienced researchers in your field

- rapid publication on acceptance

- support for research data, including large and complex data types

- gold Open Access which fosters wider collaboration and increased citations

- maximum visibility for your research: over $100 \mathrm{M}$ website views per year

At BMC, research is always in progress.

Learn more biomedcentral.com/submissions 